\title{
Against Pointillisme about Geometry
}

\author{
J. Butterfield, Oxford
}

\section{Introduction}

This paper forms part of a wider campaign: to deny pointillisme. That is the doctrine that a physical theory's fundamental quantities are defined at points of space or of spacetime, and represent intrinsic properties of such points or point-sized objects located there; so that properties of spatial or spatiotemporal regions and their material contents are determined by the point-by-point facts. ${ }^{1}$

I will first describe this wider campaign (Section 2). Then I will argue against pointillisme as regards the structure of space and/or spacetime itself (Sections 3 and 4). A companion paper (2006) argues against pointillisme in mechanics, especially as regards velocity.

I will argue that the geometrical structure of space, and/or the chronogeometrical structure of spacetime, involves extrinsic properties of points, typically properties that I shall call 'spatially extrinsic'. The main debate here is whether properties of a point that are represented by vectors, tensors, connections etc. can be intrinsic to the point; typically, pointillistes argue that they can be. After formulating this debate in Section 3, I will in Section 4 focus on Bricker's (1993) discussion. For it is an unusually thorough pointilliste attempt to relate vectors and tensors in modern geometry to the metaphysics of properties. But Bricker exemplifies a tendency I reject: the tendency to reconcile pointillisme with the fact that vectorial etc. properties seem extrinsic to points and point-sized objects, by proposing some heterodox construal of the properties in question. Thus Bricker proposes that we should re-found geometry in terms of Abraham Robinson's non-standard analysis, which rehabilitates the traditional idea of infinitesimals (Robinson 1996). I reply that once the spell of pointillisme is broken, such proposed heterodox foundations of geometry are unmotivated.

In saying this, I do not mean to be dogmatic. I of course agree that the nature of the continuum is an active research area, not only historically

1 I think David Lewis first used the art-movement's name as a vivid label for this sort of doctrine: a precise version of which he endorsed. 
Mancosu (1996, Chapters 4f.), Leibniz (2001), Arthur (2006) but also in mathematics and philosophy. Indeed there are several heterodox mathematical theories of the continuum that are technically impressive and philosophically suggestive. In this paper we will only make contact with one of them, viz. non-standard analysis, as invoked by Bricker. This is for the simple reason that all the other theories offer no support for my target, pointillisme. More precisely: so far as I know, these theories do not suggest that fundamental quantities represent intrinsic properties of points or pointsized bits of matter; because either they do not attribute such quantities to points, or they even deny that there are any points. ${ }^{2}$

But it is worth glimpsing at the outset the philosophical interest of these theories; so I here list the main ideas of some of them.

(1) Two theories that are essentially revisions of analysis (calculus) are non-standard analysis, and a different rehabilitation of infinitesimals (smooth infinitesimal analysis; McClarty 1988, Bell 1998).

(2) Two other approaches are based on the idea of a space with no points, and so are no friends of pointillisme. That is: the collection of the space's parts, ordered by parthood, has no atoms, i.e. no elements that themselves have no parts.

(i) The first is essentially a revision of measure theory, and is mainly motivated by its avoidance of the measure-theoretic paradoxes, like the BanachTarski paradox. (It was pioneered by Carathéodory (1963); for philosophical introductions, cf. Skyrms (1993), Arntzenius (2000, Section 5, pp. 201-205; 2004, Section 11); we will touch on the measure-theoretic paradoxes in Section 3.3.2, but for a full account cf. Wagon (1985).)

(ii) The second is essentially a revision of topology: topology is characterized by relations between regions taken as primitive. (Cf. Menger (1978), Roeper (1997); for a philosophical introduction, cf. Arntzenius (2004, Sections 8-10).)

Finally, three comments about the connections between, and signficance of, such theories.

(a) These theories have various connections, which this quick list does not bring out. For example, Nelson (1987) shows that a modicum of nonstandard analysis greatly simplifies a rigorous development of the theories of measure and probability.

2 Broadly speaking, the second option seems more radical and worse for pointillisme; though in such theories, the structure of a set of points is often recovered by a construction, e.g. on a richly structured set of regions. 
(b) All the theories mentioned have been developed largely without regard to applications in physics. But Arntzenius discusses the prospects for doing physics, even quantum physics, in these spaces (ibid.; and for (2i), his 2003). (Of course, within quantum physics there is a tradition of speculation about discrete space or time (Kragh and Carazza 1994): for a rigorous non-relativistic quantum theory on a discrete space that is empirically equivalent to the conventional theory, cf. Davies (2003).)

(c) As regards philosophy rather than physics, the main topic connected to the above theories is mereology: which has been discussed especially in connection with the measure-theoretic paradoxes, and (2i). Recent work includes Arntzenius and Hawthorne (2006, especially Sections II, IV) and Forrest (2004, especially Sections 3-6; 2002, especially Sections 5-10).

So I am very open to suggestions about heterodox treatments of the continuum. It is just that I find the philosophical doctrine of pointillisme an insufficient reason for rejecting the orthodox treatment. Similarly in my companion paper (2006) about mechanics; though with the difference that the proposals by the targeted authors, Tooley, Robinson and Lewis, do not invoke any well-established mathematical theory. That is: I again find pointillisme an insufficient reason for rejecting orthodoxy.

I will conduct the discussion almost entirely in the context of "Newtonian" ideas about space and time. This restriction keeps things simple: and at no cost, since both the debate and my arguments carry over to the treatment of space and time in relativistic, and even quantum, physics.

\section{The wider campaign}

As I mentioned, this paper is part of a wider campaign, which I now sketch. I begin with general remarks, especially about the intrinsic-extrinsic distinction among properties (Section 2.1). Then I state my main claims; first in brief (Section 2.2), then in more detail (Section 2.3).

\subsection{Connecting physics and metaphysics}

My wider campaign aims to connect what modern classical physics says about matter with two debates in modern analytic metaphysics. The first debate is about pointillisme; but understood as a metaphysical doctrine rather than a property of a physical theory. So, roughly speaking, it is the debate whether the world is fully described by all the intrinsic properties of all the points and/or point-sized bits of matter. The second debate is whether an 
object persists over time by the selfsame object existing at different times (nowadays called 'endurance'), or by different temporal parts, or stages, existing at different times (called 'perdurance').

Endeavouring to connect classical physics and metaphysics raises two large initial questions of philosophical method. What role, if any, should the results of science have in metaphysics? And supposing metaphysics should in some way accommodate these results, the fact that we live (apparently!) in a quantum universe prompts the question why we should take classical physics to have any bearing on metaphysics. I address these questions in my (2004: Section 2, 2006a: Section 2). Here I just summarize my answers.

I of course defend the relevance of the results of science for metaphysics; at least for that branch of it, the philosophy of nature, which considers such notions as space, time, matter and causality. And this includes classical physics, for two reasons.

First, much analytic philosophy of nature assumes, or examines, so-called 'common-sense' aspects and versions of these notions: aspects and versions which reflect classical physics, especially mechanics, at least as taught in high-school or elementary university courses. One obvious example is modern metaphysicians' frequent discussions of matter as point-particles, i.e. extensionless point-masses moving in a void (and so interacting by actionat-a-distance forces), or as continua, i.e. bodies whose entire volume, even on the smallest scales, is filled with matter. Of course, both notions arose in mechanics in the seventeenth and eighteenth century.

Second, classical physical theories, in particular mechanics, are much more philosophically suggestive, indeed subtle and problematic, than philosophers generally realize. Again, point-particles and continua provide examples. The idea of mass concentrated in a spatial point (indeed, different amounts at different points) is, to put it mildly, odd; as is action-at-a-distance interaction. And there are considerable conceptual tensions in the mechanics of continua; (Wilson (1998) is a philosopher's introduction). Unsurprisingly, these subtleties and problems were debated in the heyday of classical physics, from 1700 to 1900; and these debates had an enormous influence on philosophy through figures like Duhem, Hertz and Mach - to mention only figures around 1900 whose work directly influenced the analytic tradition. But after the quantum and relativity revolutions, foundational issues in classical mechanics were largely ignored, by physicists and mathematicians as well as by philosophers. Besides, the growth of academic philosophy after 
1950 divided the discipline into compartments, labelled 'metaphysics', 'philosophy of science' etc., with the inevitable result that there was less communication between, than within, compartments. ${ }^{3}$

Setting aside issues of philosophical method, pointillisme and persistence are clearly large topics; and each is the larger for being treatable using the very diverse methods and perspectives of both disciplines, metaphysics and physics. So my campaign has to be selective in the ideas I discuss and in the authors I cite. Fortunately, I can avoid several philosophical controversies, and almost all technicalities of physics. ${ }^{4}$

But I need to give at the outset some details about how I avoid philosophical controversy about the intrinsic-extrinsic distinction among properties, and about how this distinction differs from three that are prominent in mathematics and physics.

\subsubsection{Avoiding controversy about the intrinsic-extrinsic distinction}

My campaign does not need to take sides in the ongoing controversy about how to analyse, indeed understand, the intrinsic-extrinsic distinction. (For an introduction, cf. Weatherson (2002, especially Section 3.1), and the symposium, e.g. Lewis (2001), that he cites.) Indeed, most of my discussion can make do with a much clearer distinction, between what Lewis (1983, p. 114) dubbed the 'positive extrinsic' properties, and the rest. This goes as follows.

Lewis was criticizing Kim's proposal, to analyze extrinsic properties as those that imply accompaniment, where something is accompanied iff it coexists with some wholly distinct contingent object, and so to analyze intrinsic (i.e. not extrinsic) properties as those that are compatible with being

3 Thus I see my campaign as a foray into the borderlands between metaphysics and philosophy of physics: a territory that I like to think of as inviting exploration, since it promises to give new and illuminating perspectives on the theories and views of the two communities lying to either side of it-rather than as a no-man's-land well-mined by two sides, ignorant and suspicious of each other!

4 Though persistence is not this paper's topic, I note that among the philosophical issues my campaign avoids are several about persistence, such as: (a) the gain and loss of parts (as in Theseus' ship); (b) the relation of "constitution" between matter and object (as in the clay and the statue); (c) vagueness, and whether there are vague objects. Agreed, there are of course connections between my claims and arguments, and the various issues, both philosophical and physical, that I avoid: connections which it would be a good project to explore. But not in one paper, or even in one campaign! 
unaccompanied, i.e. being the only contingent object in the universe (for short: being lonely). Lewis objected that loneliness is itself obviously extrinsic. He also argued that there was little hope of amending Kim's analysis. In particular, you might suggest that to be extrinsic, a property must either imply accompaniment or imply loneliness: so Lewis dubs these disjuncts 'positive extrinsic' and 'negative extrinsic' respectively. But Lewis points out that by disjoining and conjoining properties, we can find countless extrinsic properties that are neither positive extrinsic nor negative extrinsic; (though 'almost any extrinsic property that a sensible person would ever mention is positive extrinsic' (1983, p. 115)).

This critique of Kim served as a springboard: both for Lewis' own preferred analysis, using a primitive notion of naturalness which did other important work in his metaphysics (Lewis 1983a); and for other, metaphysically less committed, analyses, developed by Lewis and others (e.g. Langton and Lewis 1998, Lewis 2001).

But I will not need to pursue these details. As I said, most of my campaign can make do with the notion of positive extrinsicality, i.e. implying accompaniment, and its negation. That is, I can mostly take pointillisme to advocate properties that are intrinsic in the weak sense of not positively extrinsic. So this makes my campaign's claims, i.e. my denial of pointillisme, logically stronger; and so I hope more interesting. Anyway, my campaign makes some novel proposals about positive extrinsicality: in this paper, I distinguish temporal and spatial (positive) extrinsicality; and in the companion paper against pointillisme in mechanics, I propose degrees of (positive) extrinsicality.

\subsubsection{Distinction from three mathematical distinctions}

Both the murky intrinsic-extrinsic distinction, and the clearer distinction between positive extrinsics and the rest, are different distinctions from three that are made within mathematics and physics, especially in those parts relevant to us: viz. pure and applied differential geometry. The first of these distinctions goes by the name 'intrinsic'/'extrinsic'; the second is called 'scalar'/'non-scalar', and the third is called 'local'/'non-local'. They are as follows.

(i) The use of 'intrinsic' in differential geometry is a use which is common across all of mathematics: a feature is intrinsic to a mathematical object (structure) if it is determined (defined) by just the object as given, without appeal to anything extraneous-in particular a choice of a coordinate system, or of a basis of some vector space, or of an embedding of the object into 
another. For example, we thus say that the intrinsic geometry of a cylinder is flat; it is only as embedded in $\mathbb{R}^{3}$ that it is curved.

(ii) Differential geometry classifies quantities according to how they transform between coordinate systems: the simplest case being scalars which have the same value in all coordinate systems. (Nevermind the details of how the other cases - vectors, tensors, connections, spinors etc.- transform.)

(iii) Differential geometry uses 'local' (as vs. 'global') in various ways. But the central use is that a mathematical object (structure) is local if it is associated with a point by being determined (defined) by the mathematical structures defined on any neighbourhood, no matter how small, of the point. In this way, the instantaneous velocity of a point-particle at a spacetime point, and all the higher derivatives of its velocity, are local since their existence and values are determined by the particle's trajectory in an arbitrarily small neighbourhood of the point. Similarly, an equation is called 'local' if it involves only local quantities. In particular, an equation of motion is called 'local in time' if it describes the evolution of the state of the system at time $t$ without appealing to any facts that are a finite (though maybe very small) time-interval to the past or future of $t$.

I will not spell out seriatim some examples showing that the two philosophical distinctions are different from the three mathematical ones. Given some lessons in differential geometry (not least learning to distinguish (i) to (iii) themselves!), providing such examples is straightforward work. Suffice it to make three comments, of increasing relevance for this paper.

(1) It would be a good project to explore the detailed relations between these distinctions. In particular, the mathematical distinction (i) invites comparison with Vallentyne's (1997) proposal about the intrinsic-extrinsic distinction. Besides, there are yet other distinctions to explore and compare: for example, Earman (1987) catalogues some dozen senses of 'locality'.

But in this paper and its companion, two of the various differences amongst these distinctions are especially relevant.

(2) The first is the difference between mathematical locality, (iii) above, and philosophical intrinsicality. The difference is clear for the case of instantaneous velocity. This is the main topic of my (2006); but the idea is that velocity has implications about the object at other times, for example that it persists for some time. So most philosophers say that instantaneous velocity is an extrinsic property. I agree. But emphasising its extrinsicness tends to make one ignore the fact that it is mathematically local, i.e. determined by the object's trajectory in an arbitrarily small time-interval. And in pure and 
applied differential geometry, it would be hard to over-estimate the importance of - and practitioners' preference for! — such local quantities and local equations involving them. (It is this locality that prompts me to speak of instantaneous velocity (and other local quantities) as 'hardly extrinsic'.)

(3): In this paper, we will also note the difference between being a mathematical scalar, (ii) above, and being philosophically intrinsic. Thus philosophers tend to think that any scalar quantity represents an intrinsic property of the points on which it is defined; (so that the pointilliste has only to worry about whether vectors, tensors etc. can represent intrinsic properties). But as we shall see in Section 4.4.1, that is wrong. For the scalar curvature $R$ at a point $p$ is surely extrinsic in the philosophical sense, since it gives information about the geometry of neighbourhoods of $p$. ( $R$ is also local and mathematically intrinsic; i.e. on the "intrinsic side" of all three mathematical distinctions, (i)-(iii).)

\subsection{Classical mechanics is not pointilliste, and can be perdurantist}

\subsubsection{Two versions of pointillisme}

To state my campaign's main claims, it is convenient to first distinguish a weaker and a stronger version of pointillisme, understood as a metaphysical doctrine. They differ, in effect, by taking 'point' in pointillisme to mean, respectively, spatial, or spacetime, point.

Taking 'point' to mean 'spatial point', I shall take pointillisme to be, roughly, the doctrine that the instantaneous state of the world is fully described by all the intrinsic properties, at that time, of all spatial points and/or pointsized bits of matter.

As I said in Section 2.1, my campaign can mostly take 'intrinsic' to mean 'lacking implications about some wholly distinct contingent object'; in other words, to mean the negation of Lewis' 'positive extrinsic' (i.e. his 'implying accompaniment'). But for this version of pointillisme, I will take 'intrinsic' to mean 'spatially intrinsic'. That is, attributing such a property to an object carries no implications about spatially distant objects; but it can carry implications about objects at other times. (Such objects might be other temporal parts of the given object.) So I shall call this version, 'pointillisme as regards space'.

On the other hand: taking 'point' to mean 'spacetime point', I shall take pointillisme to be, roughly, the doctrine that the history of the world is fully described by all the intrinsic properties of all the spacetime points and/or 
all the intrinsic properties at all the various times of point-sized bits of matter (either point-particles, or in a continuum). And here I take 'intrinsic' to mean just the negation of Lewis' 'positive extrinsic'. That is, it means 'both spatially and temporally intrinsic': attributing such a property carries no implications about objects at other places, or at other times. I shall call this stronger version, 'pointillisme as regards spacetime'.

So to sum up: pointillisme as regards space vetoes spatial extrinsicality; but pointillisme as regards spacetime also vetoes temporal extrinsicality.

On either reading of pointillisme, it is of course a delicate matter to relate such metaphysical doctrines, or the endurance-perdurance debate, to the content of specific physical theories. Even apart from Section 2.1's questions of philosophical method, one naturally asks, for example, how philosophers' idea of intrinsic property relates to the idea of a physical quantity. For the most part, I shall state my verdicts about such questions case by case. But one main tactic for relating the metaphysics to the physics will be to formulate pointillisme as a doctrine relativized to (i.e. as a property of) a given physical theory (from Section 2.3 onwards). Anyway, I can already state my main claims, in terms of these two versions of pointillisme. More precisely, I will state them as denials of two claims that are, I think, common in contemporary metaphysics of nature.

\subsubsection{Two common claims}

Though I have not made a survey of analytic metaphysicians, I think many of them hold two theses, which I will dub (FPo) (for 'For Pointillisme') and (APe) (for 'Against perdurantism'); as follows.

(FPo): Classical physics - or more specifically, classical mechanics—supports pointillisme: at least as regards space, though perhaps not as regards spacetime. There are two points here:-

(a) Classical physics is free of various kinds of "holism", and thereby antipointillisme, that are suggested by quantum theory. Or at least: classical mechanics is free. (With the weaker claim, one could allow, and so set aside, some apparently anti-pointilliste features of advanced classical physics, e.g. anholonomies in electromagnetism and the non-localizability of gravitational energy in general relativity: features rich in philosophical suggestions (Batterman 2003, Belot 1998, Hoefer 2000) — but not for this paper!)

(b) The concession, 'perhaps not as regards spacetime', arises from the endurance-perdurance debate. For it seems that pointillisme as regards spacetime must construe persistence as perdurance; (while pointillisme as 
regards space could construe it as endurance). And a well-known argument, often called 'the rotating discs argument', suggests that perdurance clashes with facts about the rotation of a continuum (i.e. a continuous body) in classical mechanics. So the argument suggests that classical mechanics must be understood as "endurantist". Besides, whether or not one endorses the argument, in classical mechanics the persistence of objects surely can be understood as endurance-which conflicts with pointillisme as regards spacetime.

(The considerations under (a) and (b) are usually taken as applying equally well to non-relativistic and relativistic classical mechanics: an assumption I largely endorse.)

I also think that many metaphysicians would go further and hold that:

(APe) Classical mechanics does indeed exclude pointillisme as regards spacetime: their reason being that this pointillisme requires perdurance and that they endorse the rotating discs argument. So they hold that in classical mechanics the persistence of objects must be understood as endurance, and that this forbids pointillisme as regards spacetime.

\subsubsection{My contrary claims}

I can now state the main position of my wider campaign. Namely, I deny both claims, (FPo) and (APe), of Section 2.2.2. I argue for two contrary claims, (APo) (for 'Against Pointillisme) and (FPe) (for 'For perdurantism'), as follows.

(APo): Classical mechanics does not support pointillisme.

By this I do not mean just that:

(a) it excludes pointillisme as regards spacetime.

Nor do I just mean:

(b) it allows one to construe the persistence of objects as endurance.

(But I agree with both (a) and (b).) Rather, I also claim: classical mechanics excludes pointillisme as regards space. That is: it needs to attribute spatially extrinsic properties to spatial points, and/or point-sized bits of matter. (But this will not be analogous to the kinds of "holism" suggested by quantum theory.)

(FPe) Though (as agreed in (APo)) classical mechanics excludes pointillisme as regards spacetime (indeed, also: as regards space): classical mechanics is compatible with perdurance. That is: despite the rotating discs argument, one can be a "perdurantist" about the persistence of objects in classical mechanics. The reason is that once we reject pointillisme, perdur- 
ance does not need persistence to supervene on temporally intrinsic facts. In fact, perdurantism can be defended by swallowing just a small dose of temporal extrinsicality.

So to sum up my wider campaign, I claim that:-

(APo) Classical mechanics denies pointillisme, as regards space as well as spacetime. For it needs to use spatially extrinsic properties of spatial points and/or point-sized bits of matter, more than is commonly believed.

(FPe) Classical mechanics permits perdurantism. It does not require temporally extrinsic properties (of matter, or objects), in the sense of requiring persistence to be endurance: as is commonly believed. A mild dose of temporal extrinsicality can reconcile classical mechanics with perdurance.

To put the point in the philosophy of mind's terminology of 'wide' and 'narrow' states, meaning (roughly) extrinsic and intrinsic states, respectively: I maintain that classical mechanics:

(APo) needs to use states that are spatially wide, more than is commonly believed; and

(FPe) does not require a specific strong form of temporal width, viz. endurance. With a small dose of temporal extrinsicality, it can make do with temporally quite narrow states-and can construe persistence as perdurance.

\subsection{In more detail ...}

So much by way of an opening statement. I will now spell out my main claims in a bit more detail: first (APo), and then, more briefly, (FPe).

\subsubsection{Four violations of pointillisme}

I will begin by stating pointillisme as a trio of claims that apply to any physical theory; and making two comments. Then I list four ways in which (chrono)-geometry and classical mechanics violate pointillisme: three will form the main topics of this paper and its companion.

The trio of claims is as follows:

(a) the fundamental quantities of the physical theory in question are to be defined at points of space or of spacetime;

(b) these quantities represent intrinsic properties of such points;

(c) models of the theory-i.e. in physicists' jargon, solutions of its equations, and in metaphysicians' jargon, possible worlds according to the theory-are fully defined by a specification of the quantities' values at all such points. 
So, putting (a)-(c) together: the idea of pointillisme is that the theory's models (or solutions or worlds) are something like conjunctions or mereological fusions of "ultralocal facts", i.e. facts at points.

Two comments. First: the disjunction in (a), 'at points of space or of spacetime', corresponds to Section 2.2's distinction between pointillisme as regards space, and as regards spacetime. Nevermind that it does not imply the convention I adopted in Section 2.2, that pointillisme as regards spacetime is a stronger doctrine: since it vetoes temporally extrinsic properties, as well as spatially extrinsic ones. The context will always make it clear whether I mean space or spacetime (or both); and whether I mean spatially or temporally extrinsic (or both).

Second: Though I have not made a systematic survey, there is no doubt that pointillisme, especially its claims (a) and (b), is prominent in contemporary metaphysics of nature, especially of neo-Humean stripe. The prime example is David Lewis' metaphysical system, which is so impressive in its scope and detail. One of his main metaphysical theses, called 'Humean supervenience', is a version of pointillisme: I will return to it in Section 3.2.

When we apply (a)-(c) to classical mechanics, there are, I believe, four main ways in which pointillisme fails: or, more kindly expressed, four concessions which pointillisme needs to make. The first three violations (concessions) occur in the classical mechanics both of point-particles and of continua; the fourth is specific to continua. And the first two are addressed in this paper; the third is discussed in the companion paper (2006).

(1) The first violation is obvious and minor. Whether matter is conceived as point-particles or as continua, classical mechanics uses a binary relation of occupation, '... occupies ...', between bits of matter and spatial or spacetime points (or, for extended parts of a continuum: spatial or spacetime regions). And this binary relation presumably brings with it extrinsic properties of its relata: it seems an extrinsic property of a point-particle (or a continuum, i.e. a continuous body) that it occupy a certain spatial or spacetime point or region; and conversely.

Agreed, there is more to be said about this claim (as always in philosophy!): both about (a) the connections between the intrinsic-extrinsic distinction among properties and the classification of relations, and (b) how the individuation of spatial or spacetime points or regions might depend on matter (the "relational conception" of space or spacetime). I will discuss (a) and (b), albeit briefly in Sections 3.3. But anyway, I will there endorse the claim. That is: the concession will remain in force: the pointilliste about 
classical mechanics should accept this binary relation of occupation, and the modicum of extrinsicality it involves.

(2) Classical mechanics (like other physical theories) postulates structure for space and/or spacetime (geometry or chrono-geometry); and this involves a complex network of geometric relations between, and so extrinsic properties of, points. This concession is of course more striking as regards space than time: three-dimensional Euclidean geometry involves more structure than does the real line. This will be the main topic of this paper.

(3) Mechanics needs of course to refer to the instantaneous velocity or momentum of a body; and this is temporally extrinsic to the instant in question, since for example it implies the body's existence at other times. (But it is also local in the sense of (iii), Section 2.1.2.) So this second violation imposes temporal, rather than spatial, extrinsicality; i.e. implications about other times, rather than other places.

This is the main topic of Butterfield (2006). But I should stress here that this third violation is mitigated for point-particles, as against continua. For a pointilliste can maintain that the persistence of point-particles supervenes on facts that, apart from the other violations (i.e. about 'occupies' and (chrono)geometry), are pointillistically acceptable: viz. temporally intrinsic facts about which spacetime points are occupied by matter. In figurative terms: the void between distinct point-particles allows one to construe their persistence in terms of tracing the curves in spacetime connecting points that are occupied by matter. I develop this theme in my (2005). On the other hand: for a continuous body, the persistence of spatial parts (whether extensionless or extended) does not supervene on such temporally intrinsic facts. This is the core idea of the rotating discs argument, mentioned in Section 2.2.2.

To sum up: the rotating discs argument means that pointillisme fits better with point-particles than with continua. To put the issue in terms of Section 2.2's two forms of pointillisme: the strong form of pointillisme, pointillisme as regards spacetime, fails for the classical mechanics of continua, even apart from the other concessions mentioned.

(4) Finally, there is a fourth way that the classical mechanics of continua violates pointillisme: i.e., a fourth concession that pointillisme needs to make. Unlike the rotating discs argument, this violation seems never to have been noticed in recent analytic metaphysics; though the relevant physics goes back to Euler. Namely, the classical mechanics of continua violates (the weaker doctrine of) pointillisme as regards space, because it must be formulated in terms of spatially extended regions and their properties and rela- 
tions. But in this paper, I set this fourth violation aside entirely; my (2006a) gives details.

So to sum up these four violations, I claim (APo): classical mechanics violates pointillisme. This is so even for the weaker doctrine, pointillisme as regards space. And it is especially so, for the classical mechanics of continua rather than point-particles.

\subsubsection{For perdurantism}

I turn to Section 2.2.3's second claim, (FPe): that once pointillisme is rejected, perdurantism does not need persistence to supervene on temporally intrinsic facts, and can be defended for classical mechanics provided it swallows a small dose of temporal extrinsicality.

About (FPe) I can be much briefer, since this paper will not need details. I will just identify this small dose: it is the extrinsicality of the third violation of pointillisme above-in particular, the presupposition of persistence by the notion of a body's instantaneous velocity. Thanks to the rotating discs argument, 'body' here means especially 'point-sized bit of matter in a continuum'; since for point-particles we can construe persistence as perdurance without having to take this dose. Elsewhere (2004, 2004a, 2006) I argue that for a "naturalist" perdurantist, this dose is small enough to swallow.

\section{Can properties represented by vectors be intrinsic to a point?}

\subsection{Prospectus}

I turn to the geometrical structure of space, and/or the chrono-geometrical structure of spacetime. I will argue that this structure involves extrinsic properties, especially spatially extrinsic properties. I will undertake three specific tasks, in Sections 3.2, 3.3, 4 respectively.

In Section 3.2, I present Lewis' version of pointillisme. Though this version is in some ways logically stronger than I need, it is important to present it. Not only has it been a focus of recent metaphysical discussion; it is also needed for Section 4.

In Sections 3.3 and 4, I argue in detail that pointillisme needs to be qualified to accommodate the structure of space and/or spacetime. I think the need for this qualification is uncontentious; in particular, it is agreed by 
Lewis. But how exactly to state the qualification is a matter that is both important and unresolved.

It is important for three reasons. First, all physical theories of course appeal to space (and/or spacetime). Second, they all represent the properties that encode the structure of space or spacetime, with mathematical entities such as vectors, tensors, connections etc. So the question arises: can properties that are so represented be intrinsic to a point? The third reason is taken up in the companion paper (2006): it is that physical theories also represent the other properties they mention, i.e. properties of matter such as velocity, momentum etc., by such mathematical entities as vectors, tensors, connections etc.

So the question - can properties represented by vectors, tensors etc. be intrinsic to a point? - is at the centre of this paper (and its companion). First, in Section 3.3, I will lead up to this question by discussing, in a broadly metaphysical way, how to represent the structure of space or spacetime. (I will concentrate on the notion of length, and so on space rather than spacetime; but this discussion carries over intact to the case of spacetime.) Once the question is posed, Section 4 addresses it in detail, using as a foil Bricker's (1993).

As I said in Section 1, Bricker's paper illustrates how strongly some contemporary metaphysicians are attracted by pointillisme. For recognizing that they must accept vectorial properties in physical theories, and that these seem not to be intrinsic to points, they propose to save pointillisme by advocating a heterodox construal of the property. Thus in Section 4, Bricker will construe the metric tensor of differential geometry in terms of non-standard analysis. (And in the companion paper, Tooley and others will construe instantaneous velocity as intrinsic.) My own view will of course be that there is no need for such heterodoxy: instead, we can and should reject pointillisme.

My discussion will be simplified by a restriction. I will consider only properties represented by vectors and tensors, which I will for short call vectorial properties and tensorial properties: not those represented by other mathematical entities such as connections. This restriction will be natural, in that:

(i) vectors and tensors are about the simplest of the various mathematical entities that physical theories use to represent properties and relations-so they are the first case to consider;

(ii) the restriction is common in the literature; in fact most of the authors I discuss (here and in the companion paper) consider only vectorial properties. 


\subsection{Humean supervenience}

I will assume familiarity with the main ideas of Lewis' metaphysical system, above all his notions of possible world and natural property. Central to this system is Lewis' version of pointillisme, which he says (1994, p. 494) is inspired by classical physics. He calls this doctrine 'Humean supervenience'. It is stronger than pointillisme as defined in Section 2.3, in that it is not relative to a theory. Roughly, it is relative to a possible world; (of course a metaphysician like Lewis who accepts the idea of a law of nature can link relativizations to a theory and to a possible world using the idea of the "complete" theory of a world, say as an axiomatization of all its laws of nature). And Lewis claims that it holds at the actual world.

The idea of Humean supervenience is that all truths supervene on truths about matters of local particular fact: where 'matters of local particular fact' is to be understood in terms of Lewis' metaphysics of natural properties, with the properties having spacetime points, or perhaps point-sized bits of matter, as instances. Thus he writes that Humean supervenience

... says that in a world like ours, the fundamental relations are exactly the spatiotemporal relations: distance relations, both spacelike and timelike, and perhaps also occupancy relations between point-sized things and spacetime points. And it says that in a world like ours, the fundamental properties are local qualities: perfectly natural intrinsic properties of points, or of point-sized occupants of points. Therefore it says that all else supervenes on the spatiotemporal arrangement of local qualities throughout all of history, past and present and future. (1994, pp. 225-226. $)^{5}$

Humean supervenience, so defined, is not widely believed-few philosophers sign up to all the notions deployed in its statement. But it has been a natural focus of metaphysicians' attention in the last twenty years, not least because Lewis has been the pre-eminent neo-Humean. In the literature, we can distinguish three broad groups of topics:

(i) Issues about whether to analyse law, causation, chance etc., and "higher-level“ concepts about mind and language, in terms of the notions of Lewis' framework. Lewis (1986, p. xi-xiv) sketches how his work on all these topics provides a "battle-plan": i.e. roughly, a sequence of supervenience claims for these concepts. More generally, much literature of neo-

5 Cf. also his (1986, pp. ix-x). 
Humean stripe is concerned with how truths using familiar central concepts of common-sense knowledge and belief-concepts such as law, causation, the persistence of objects and mental and semantic concepts, such as belief and reference-might supervene on a basis acceptable to Humeans, though perhaps not exactly the basis proposed by Lewis. (For example: for laws, cf. Earman and Roberts (2006).) And some of these truths pose a challenge in that they seem not to thus supervene; cf. (iii) below.

(ii) General metaphysical issues about the notions of Lewis' framework, in particular possible worlds and natural properties, and/or about related notions. For example, one well-known issue is: can possible worlds and natural properties be construed less "realistically" than Lewis proposes (e.g. Taylor 1993), and yet do the philosophical work they are meant to do? More relevant to us will be Lewis' view of the intrinsic-extrinsic distinction, viz. that it can be analysed in terms of perfectly natural properties; (details in Section 4.2). But as discussed in Sections 2.1.1 and 2.2.1, I can for the most part use only a much clearer distinction, viz. between the positive extrinsic properties and the rest, sub-divided in terms of temporal and spatial implications (or lack of them).

(iii) Direct threats to Humean supervenience. There are two main examples. First, chance; which Lewis addresses in detail in (1986, pp. xiv-xvi, 121-131), and to his greater satisfaction in (1994). Second, persistence. For Lewis as a Humean wants to be perdurantist, as well as pointilliste in the sense of Humean supervenience: this means that he faces the rotating discs argument.

In this paper, I can set aside all of (i) and (iii), and all of (ii) except for the intrinsic-extrinsic distinction.

\subsection{Accommodating space and spacetime}

\subsubsection{An agreed concession}

As I mentioned, pointillisme's need to accommodate the structure of space and/or spacetime is agreed by all parties: in particular, by Lewis. In both the quotations above, Lewis includes relations of spatiotemporal distance (spacelike and timelike) in the supervenience basis. So his Humean supervenience is not so pointilliste, at least as regards the structure of space and/or spacetime, as it might at first seem.

But no doubt even the most ardent pointilliste will find the inclusion sensible. That is: no one will hold that the structure of space and/or spacetime, 
in particular spatial and/or spatiotemporal metrical relations, is to supervene on intrinsic properties of points. ${ }^{6}$

The natural thing to say is, instead, that the points and these metrical relations (and maybe also some spatiotemporal but non-metrical relations) form collectively a background, or canvas, on which other physical quantities taking various values get "painted". And it is to these latter that pointillisme's doctrines are to apply.

But there is no consensus (indeed, not much discussion) about how precisely to state the concession. More's the pity, since apart from the concession's own importance, it leads to the more general question (taken up in the following Subsections) how pointillisme can accommodate vectorial and tensorial properties.

I begin with a preliminary issue. The concession obviously relates to the debate between relationist and substantivalist views of space and spacetime; and though I will not pursue this debate, I should register that this concession is not meant to prejudge it. There are two points here.

(i) Though I spoke like a substantivalist, about a canvas of points, with various metrical and non-metrical relations between them, it is safe to assume that a relationist would appeal to similar relations holding between items of their preferred ontology, i.e. bodies. (I set aside whether Leibniz's monads with only their intrinsic properties might be enough to subvene all spatial and spatiotemporal facts!)

(ii) Similarly, my talk of a canvas of points, with metrical and non-metrical relations between them, was not meant to deny that the metric (or the other relations) could be dynamical, i.e. influenced by matter, in the way they are in general relativity.

So our question is what exactly metrical (and other geometrical) structures require. As physical geometry has developed in the last two hundred years, these requirements have not only become subtler but have also become bound up with other properties and relations, especially of matter, in

6 Here I recall this paper's restriction to classical mechanics. So I of course set aside speculations in quantum gravity that classical spacetime structure emerges somehow from a "quantum pre-geometry": speculations which, I agree, might have this structure emerge from (or even supervene on) intrinsic properties of some pointlike objects. But I doubt it: most schemes for quantum pre-geometry are thoroughly "relational" rather than pointilliste. For surveys of such speculations, cf. e.g. Monk (1997), Butterfield and Isham (1999, Sections 1-4; 2001, Section 5); for a brief discussion in relation to Lewis' Humean supervenience, cf. Oppy (2000, p. 88, 91-94). 
ways which threaten pointillisme. This issue will extend to Section 4. But let us start by raising the issues involved in as simple a context as possible: the length of a straight line in elementary geometry.

\subsubsection{The length of a line}

The length of a line is the topic of a venerable paradox. The length of a straight line should surely be the sum of the lengths of a decomposition, i.e. an exhaustive set of mutually non-overlapping parts; and it seems legitimate to take as these parts the line's constituent points; but the length of each point is zero, and the sum of all these zeroes is presumably (though a continuously large sum) zero-what else could it be? So the length of the line is zero!

I stated this paradox in its most familiar form, as about summing lengths. But of course it can also be stated in philosophical terms, as about supervenience: the length of a line surely does not supervene on the lengths of its points, on pain of being zero. That is no doubt why, as discussed in Section 3.3.1, no pointilliste holds that lengths (or other metrical properties of lines, or indeed metrical relations between points) supervene on intrinsic properties of points. ${ }^{7}$

This paradox is of course one of many that eventually prompted the development of measure theory. And as noted in (2i) and (c) of Section 1, measure theory invites philosophical scrutiny because (i) it has some wellnigh paradoxical results of its own, like the Banach-Tarski paradox, and (ii) it is connected to mereology. But I shall not need details about these topics. I only need to present:

(a) a philosophical reply to this paradox; though it does not block the paradox, it introduces an important metaphysical trichotomy among relations;

(b) the main idea of the technical measure-theoretic reply to this paradox.

3.3.2.A The philosophical reply. The philosophical reply is just that length is a property of the line as a whole, where 'line as a whole' can be taken to mean either the set, or the mereological fusion, of its points (or of

7 There is of course a similar failure of supervenience for an extensive quantity such as mass, applied to a continuous body. For the point-sized parts of such a body have zero mass, so that the mass of the body is not the (uncountable) sum of the masses of those parts. Hawthorne notes this, while assessing how Lewis' metaphysics treats quantities (2006); and we will return to it in Section 4.6. 
its extended parts). That is, the length does not supervene on the properties of the points (or other parts). This is surely true, so far as it goes. But it is not enough to block the paradox, since it does not pinpoint what is wrong with the premise that the length of a straight line is the sum of the lengths of an exhaustive set of mutually non-overlapping parts. (The technical reply will do this.)

However, this reply prompts a trichotomy among relations corresponding to the intrinsic-extrinsic dichotomy among properties: a trichotomy that will be useful in what follows. Lewis states the trichotomy clearly (1983a, p. 26 fn. 16; 1986a, p. 62); and I shall adopt his proposed terminology (which has become widespread). Though he explains it in terms of his preferred understanding of intrinsic and extrinsic properties (viz. defined in terms of his natural properties), the trichotomy can be explained in the very same words, using other understandings of intrinsic and extrinsic. In particular, it can be thus explained using Section 2.1.1's suggested understanding of 'extrinsic' as 'positive extrinsic' and 'intrinsic' as 'not positive extrinsic'; (or using Lewis' "second favourite" analysis developed by Langton and him (1998, p. 129)). The trichotomy also uses the idea of the mereological fusion, or composite, of objects: an idea I am happy to accept, and for which there is a powerful argument (Lewis 1986a, pp. 212-213, developed by Sider 2001, pp. 121-139).

(1) An internal relation is determined by the intrinsic properties of its relata. So if $x R y$, and $x^{\prime}$ matches $x$ in all intrinsic properties, and $y^{\prime}$ matches $y$ in all intrinsic properties, then we must have $x^{\prime} R y^{\prime}$. So any relation of similarity or difference in intrinsic respects is internal; for example, if height is an intrinsic property, then 'being taller than' is an internal relation.

(2) On the other hand, there are relations, notably relations of spatiotemporal distance, that are not internal, but do supervene on the intrinsic nature of the composite (mereological fusion) of the relata. Thus suppose $x$, $y$ are point-particles 1 metre apart. Then it seems reasonable to say both of the following:-

(i) There could be point-particles $x^{\prime}, y^{\prime}$ that intrinsically match $x$ and $y$ respectively, and that are 2 metres apart-so that distance is not internal. But on the other hand:

(ii) Any object intrinsically matching the fusion or composite of $x$ and $y$ would have two parts intrinsically matching $x$ and $y, 1$ metre apart. Accordingly, Lewis calls relations that supervene on the intrinsic nature of the fusion of the relata, external; and he takes (ii) to show that spatiotemporal relations are external. 
(3) Finally, there are relations that do not supervene even on the intrinsic nature of the composite of the relata; i.e. relations that are neither internal nor external. Lewis' example is the relation having the same owner: $x$ and $y$ could intrinsically match $x^{\prime}$ and $y^{\prime}$ respectively, and their composites might also match; and yet $x$ and $y$ might have the same owner, while $x^{\prime}$ and $y^{\prime}$ do not. But more relevant to us than ownership, geometry and mechanics provide many examples of such relations. The objects $x, y, x^{\prime}, y^{\prime}$ could be solid bodies, again with each pair, $x, x^{\prime}$ and $y, y^{\prime}$, intrinsically matching, and the composites $x+y, x^{\prime}+y^{\prime}$ also matching-and yet the centre of mass of $x$ and $y$ might be a certain distance from a body of some kind $Z$, while the centre of mass of $x^{\prime}$ and $y^{\prime}$ is not.

3.3.2.B The technical reply. The technical reply to the paradox of length comes from measure theory. It blocks the paradox by denying the premise that the length of a straight line is the sum of the lengths of any decomposition (exhaustive set of mutually non-overlapping parts) of the line. It upholds this only for certain decompositions. The main idea is to consider only decompositions containing points and intervals, and to accept the additivity of length for at most denumerably large sums. These ideas give a rich theory which can be extended to cover area and volume, as well as length; and which underpins the theory of integration.

But we do not need further details of measure theory. For us the point is that, even in the elementary geometry of Euclidean space $\left(\mathbb{R}, \mathbb{R}^{2}\right.$ etc.), we cannot say all that is true in terms just of intrinsic properties of points. For we need to assign lengths to spatial intervals. And - to use Lewis' terms- the length of an interval is surely not an internal relation between the interval's end-points, since any two points seem to match in intrinsic properties. Besides, the length of an interval does not seem to be an internal relation between all the interval's uncountably many points, since: (i) the points of any two intervals seem to match pairwise in intrinsic properties, and (ii) additivity of length fails for an uncountable set.

On the other hand, it seems reasonable to say, as Lewis does, that:

(a) intervals are composites or fusions of their points; and

(b) intervals matching in their intrinsic properties are congruent; (cf. (2) (ii) above).

If we say (a) and (b), then it follows that the length of a straight interval is an external relation among the interval's points. (So far, this is a relation of uncountable polyadicity: the next Subsection will ask whether the relation can 
be taken to be just a dyadic relation between the interval's two end-points.)

To sum up: to describe length, even the length of a straight interval in Euclidean geometry, pointillisme must concede that it needs to go beyond intrinsic properties of points, and even relations that are internal in Lewis' sense. But so far, it seems (cf. (2)(ii) in Section 3.3.2.A and (b) above) it can manage with what Lewis calls external relations. ${ }^{8}$

\subsubsection{Accommodating more geometry}

The further development of geometry, including more general geometries than the Euclidean line or plane, reinforces the point that we cannot say all that is true in terms just of intrinsic properties of points. But as we shall see, it is doubtful that we can manage with just Lewis' category of external relations. That is, it is doubtful that all pointillisme needs to do, in order to accommodate spatial and spacetime structure, is to admit the network of external relations of spatial and spatiotemporal distance.

Talk of 'spatial and spatiotemporal relations' tends to suggest that space or spacetime is a metric space, in the usual mathematical sense that (given a unit of length) there is a real-valued function on pairs of points: to any pair of points $x, y$ is assigned a distance $d(x, y) \in \mathbb{R}$. (So each real number determines a binary relation on points; and for a relativistic spacetime, $d$ need not be positive-definite.) But the development of geometry and physics has shown that this is much too limited a conception of spatial (or spatiotemporal) structure. One needs to distinguish various subtly related levels of structure: for example, geometers distinguish topological structure, differential structure, metrical structure, and many more. Besides, most of these kinds of structure include definitions of irreducibly global features of the space concerned; for example, a space can have the global topological feature of being simply connected, i.e. such that all closed curves can be continuously deformed to a point.

8 Incidentally, returning to the original paradox of length: the practice of measure theory seems indifferent between the following options (and surely metaphysicians can be as well):

a) to allow $a b$ initio that each point has a length, viz. zero; and avoid paradox by denying uncountable additivity of length;

b) to ascribe length primarily only to sets of points in a certain well-behaved family of sets.

But the technical need for the family to have certain closure properties is likely to lead to singleton sets of points being included - as in the usual choice of family, the Borel sets. 
But here I will focus only on "local" metrical structure. ${ }^{9}$ Even without developing the formal details (which go back to Gauss and Riemann), we will be able to see that geometry (and more generally physics) needs to attribute to points both vectorial and tensorial properties - raising the question, pursued in the next Subsection, whether pointillisme can accommodate such properties.

Gauss and Riemann proposed that we take as the primary notion, the length of a curve between two points; (so since a pair of points is in general connected by infinitely many curves, any such pair is associated with infinitely many lengths, not just one). This proposal is adopted by modern spacetime theories, in particular by the most successful such theory, general relativity. So an advocate of pointillisme (or some similar doctrine of local supervenience, such as Humean supervenience) would do well to formulate their doctrine so as to incorporate, or at least allow for, this proposal.

At first sight, it seems that the pointilliste can manage just fine. She only needs to apply Lewis' idea of external relations (or perhaps, some similar notion), not to the endpoints of a straight interval or to all the points of a straight interval, but to all the points of an arbitrary curve. Thus she can take an arbitrary curve as the fusion of its points, and the length of the curve as an external relation, albeit of uncountable polyadicity, among the points: an external relation which determines an intrinsic property of the fusion. I presume that Lewis, who was well aware of the Gauss-Riemann conception of geometry, would have said this. That is, he would have taken 'spatiotemporal distance relations' in his definition of Humean supervenience to allow for this conception-and not to be committed to the idea of a metric space. $^{10}$

Besides, the structure required to define the lengths of all curves is given "locally" in a way that at first sight seems congenial to a pointilliste. In particular, it seems that the pointilliste does not need to postulate continuously many external relations, each of uncountable polyadicity, one for each congruence-class of curves. For:

9 I put 'local' in scare-quotes, since I here intend a vaguer meaning than that of (iii) of Section 2.1.2. But in what follows, the meaning will always be clear from the context. Note also that though I will discuss only space, all I say carries over intact to spacetime.

10 But Lewis seems never to have pursued the question exactly what relations he should propose as fundamental for modern geometry and topology; and we shall shortly see trouble for him, as for other pointillistes. 
(i) measure theory can be extended to apply to this kind of geometry, so that the length of a curve is the sum of the lengths of a countable decomposition of it;

(ii) similarly, calculus can be extended to apply to this kind of geometry, so that the length of a curve is given by an integral along it -intuitively, an uncountable sum of infinitesimal contributions one for each infinitesimal element of the curve.

These features, (i) and (ii), reflect the fact that the Gauss-Riemann conception of metrical structure presupposes topological and differential structure: which make sense, respectively, of the notions of continuous function, and differentiable function.

But there is a devil in the details. The details of how to define the length of a curve require us to attribute vectors and tensors to a point. A bit more precisely: we need to attribute:

(i) to any point on any curve, the tangent vector to the curve at that point; and so

(ii) to any point, the set of all such tangent vectors at it (which form a vector space, called the tangent space);

(iii) to any point, a metric tensor which maps pairs of tangent vectors at the point to real numbers-generalizing the elementary scalar product of two vectors. (More details about (i)-(iii) in the next Subsection.)

Thus the pointilliste has to face-already in geometry, even before considering physics' description of matter - the question announced in Section 3.1: can a property represented by a vector or a tensor be intrinsic to a point?

\section{Accommodating tangent vectors and the metric tensor}

\subsection{Bricker and others}

As I announced in Section 3.1, my main effort in this Section will be to report and criticise Bricker's (1993) discussion of this question, for metrical properties. I choose him for two reasons.

(i) His paper is an unusually thorough and perceptive attempt to relate vectors and tensors, as they are treated in modern geometry, to the modern metaphysics of properties. So it repays detailed scrutiny.

(ii) His paper illustrates the tendency that, as I said at the end of Section 3.1, I want to reject: the tendency of some contemporary metaphysicians to reconcile pointillisme with physical theories' use of vectorial properties, 
which seem extrinsic to points, by proposing some heterodox construal of the properties in question. Bricker proposes that in order to understand space's or spacetime's metrical structure as intrinsic, we should appeal to non-standard analysis. I will deny this heterodoxy: instead, we can and should reject pointillisme.

But before going into the details of Bricker's discussion, I should register that other metaphysicians have also addressed our question; though (so far as I know) more briefly and with less attention to technicalities than Bricker. (Besides, they are not all attracted by pointillisme, or by the above tendency.)

For example, Robinson maintains that the directionality of a vector forbids it from representing an intrinsic property: "direction seems to me an inherently relational matter" (1989, p. 408). And he would presumably say the same about tensorial properties. (His paper is about the rotating discs argument; I discuss its proposals in 2006.) Robinson gives an argument for this, using Lewis' notion of duplicates, i.e. objects that share all their intrinsic properties. He also credits Lewis for the argument; so presumably Lewis himself thought at the time (ca. 1988) that vectorial properties could not be intrinsic. The argument combines two intuitions:

(a) It seems that a vectorial property could not be instantiated in a zerodimensional world consisting of a single point; though since arbitrarily close points define a direction, there is of course no lower limit to the "size" of a world in which a point instantiates a vectorial property.

(b) But it also seems that, since a point in an extended world that instantiates a vectorial property is indeed a point, it could have a duplicate that existed on its own, i.e. was the only object in its world.

Taken together, (a) and (b) imply that duplicate points might differ in their vectorial properties; so that any such property is not intrinsic. ${ }^{11}$

But for anyone who is attracted by pointillisme, and is aware of physical theories' use of vectorial properties, this is a very uncomfortable conclusion. Lewis himself is a case in point. Indeed, he seems to have come round to

11 Other metaphysicians also maintain that vectorial properties are extrinsic to points. For example, Black (2000, p.103) holds that vectorial properties can be intrinsic only for the special case of vectors on a manifold with a flat connection; i.e. roughly, a manifold in which there is a unique preferred way to compare vectors located at different points. (His discussion is briefer than Robinson's; since the topic is less relevant to his paper's main aims, than it is to Robinson's.) And Zimmerman (1998, p. 277-278) and Oppy (2000, pp. 79-82) are similarly inclined; though they also discuss sympathetically the opposing view. 
believing that vectors can represent intrinsic properties of points, sometime between ca. 1988 and ca. 1993. For in a discussion of Humean supervenience $(1994$, p. 474), he says he is inclined to think that vectorial properties are, or at least can be, intrinsic: "any attempt to reconstrue them as relational properties seems seriously artificial”. But, so far as I know, that is all Lewis says by way of defending the idea; (though in his (1999) he used the idea to try and reply to the rotating disc argument-unsuccessfully I maintain (2006)). In any case, I now turn to Bricker's extended struggle to avoid the uncomfortable conclusion.

\subsection{Bricker's three claims about metrical structure}

\subsubsection{Bricker's metaphysical framework}

Bricker's (1993) overall aim is metaphysical understanding of spatial (or spatiotemporal) relations. He adopts a metaphysical framework very close to Lewis'-with of course all due acknowledgement (1993, pp. 273-5). The ingredients we need are:-

(i) He speaks of possible worlds and perfectly natural properties and relations. He applies mereology freely to points of space and spacetime; (in fact, substantivalism about space and spacetime is widespread among analytic metaphysicians). And so he takes worlds and parts of worlds as possibilia.

(ii) He says that any two possibilia $X, Y$ are duplicates iff there is a one-toone correspondence between their parts that preserves all perfectly natural properties and relations. He calls any such correspondence an $(X, Y)$ counterpart relation, and corresponding parts are $(X, Y)$-counterparts of each other. (So in this Subsection, 'counterpart' is tied to 'duplicate' and so will not have the usual Lewisian connotations of allowing vagueness and extrinsicness.)

(iii) He says that a property is intrinsic iff any two duplicates both have it or both lack it. (Otherwise the property is extrinsic.) It follows that:

(a) the class of all possibilia is partitioned by the equivalence relation of being duplicates; and

(b) an intrinsic property corresponds to a union of cells of this partition; and

(c) all perfectly natural properties are intrinsic.

He extends the notion of intrinsic to relations by saying that a relation is intrinsic iff it is either internal or external in the senses of Lewis (defined in Section 3.3.2.A); otherwise the relation is extrinsic. 
(iv) He assumes (following Lewis 1986a, pp. 86-92) a principle of recombination for spatial or spacetime points. This is a principle of modal plenitude, inspired by a Humean denial of necessary connections between distinct existences: "anything can follow anything". Stated for spatial points, it holds: for any points $p$ and $q$, perhaps from spaces of different worlds, there is a world whose space is a duplicate of the space of $p$, except that it contains a duplicate of $q$ where the duplicate of $p$ would be (1993, p. 290); and similarly for spacetime.

I do not endorse this framework. But in discussing Bricker, I will use it (and a variant of it considered by him). Though it would be a good project to ascertain how well Bricker's arguments fare under a different framework (in particular, under weaker assumptions about the intrinsic-extrinsic distinction), it is not a project for this paper. Here it must suffice to note that if we used my distinction between positive extrinsics and the rest, advocated in Section 2.1.1, the main points of my critique of Bricker below, would carry over intact. But I shall not spell this out point by point, from now on. I just note here that:

(a) Since my distinction takes 'intrinsic' to mean 'not positively extrinsic', it yields more intrinsic properties than does Bricker's (or Lewis') framework; and so a logically stronger notion of duplicatehood as sharing of all intrinsic properties.

(b) Bricker's argument for the spacetime metric being extrinsic to points (Section 4.3) remains valid on my distinction's construal of 'extrinsic' as 'positive extrinsic'. For Bricker's argument implicitly appeals to positive extrinsicality.

(c): My anti-pointilliste reply to Bricker (Section 4.6) is unaffected by adopting my distinction.

Bricker goes on to connect his framework with the Gauss-Riemann conception of distance, as endorsed by general relativity. His discussion includes aspects (1993, pp. 275-286) which we can skip, in particular: (a) a comparison with two other conceptions of distance (which he dubs the 'naive' and 'intrinsic' conceptions); and (b) a discussion of how the principle of contact-action (denial of action-at-a-distance) bears on the the Gauss-Riemann conception. Setting these aside, I read Bricker as connecting his metaphysical framework with the Gauss-Riemann conception, as follows. He assumes that:

(i) the perfectly natural properties and relations, that are instantiated at a possible world that has laws of nature, figure in that world's laws (however the notion of a law of nature is to be analysed); 
(ii) general relativity is a logically possible theory, giving the gravitational and metrical laws of some possible worlds;

(iii) general relativity can be "formulated locally"; which is taken to imply, as regards metrical structure, formulated in terms of local metrical relations. Taking these assumptions together, he concludes that the property of having such-and-such a local metric tensor is a perfectly natural property, and is instantiated at points in general relativistic worlds.

So far, Bricker is in a position like the one we articulated at the end of Section 3.3, in which the pointilliste seemed well able to manage local metrical structure. Bricker also notes (as we did) that since even topology brings in irreducibly global properties of space like being simply connected, there can be no sweeping supervenience of the global on the local. So he formulates a doctrine he calls Einsteinian supervenience, on analogy with Lewis' Humean supervenience: there is "a manifold of spacetime points ... and a distribution of perfectly natural local properties (including local metrical properties) over those points; all else supervenes on that" (1993, p. 288).

\subsubsection{Bricker's three claims}

Bricker then notices what I called 'the devil in the details', i.e. the fact that local metrical structure attributes vectorial and tensorial properties to points; and he goes on to address the question whether such properties are intrinsic, in terms of his metaphysical framework (1993, pp. 288f.). He argues for the following three claims (in order, with my added mnemonic labels).

(MetrExtr) The metric tensor, as standardly conceived in differential geometry, represents an extrinsic property of a point.

(VetoExtr) The obvious (and anti-pointilliste) response to the conflict between this and the metric being perfectly natural-viz. that some but not all perfectly natural properties are intrinsic-does not work. For, Bricker argues, it clashes with the Humean principle of recombination for spacetime points. That is, Bricker rejects this response as engendering necessary connections between distinct existences, viz. a point and its surrounding space.

So Bricker claims we do better to revise our conception of the metric tensor, as follows.

(Heterodox) We should take the metric to represent an intrinsic property of an infinitesimal neighbourbood of a point. Bricker cites Robinson's non-standard analysis as justifying taking such neighbourhoods as genuine 
mathematical objects, rather than as a facon de parler for calculus' standard notion of limit as “ $\forall \exists \forall$ ” (e.g. for a real sequence $\left\{a_{n}\right\}: \forall \varepsilon>0 \exists N \forall m, n>$ $\left.N\left|a_{m}-a_{n}\right|<\varepsilon\right)$.

So Bricker's overall conclusion is radical: that in order to save pointillisme, we should revise the foundations of differential geometry. In the next three Subsections, I will report his arguments for (MetrExtr) to (Heterodox). Then in the last Subsection (Section 4.6), I will deny his conclusion. Since I hold no brief for pointillisme, I see no reason to pay his price of revising the foundations of differential geometry.

\subsection{The standard metric is extrinsic}

Bricker's argument for (MetrExtr) - the metric tensor, as standardly conceived, represents an extrinsic property of a point-is not absolutely precise. But it uses more technicalities about local metrical structure than I have introduced so far, in particular differential geometry's idea that the tangent vectors at a point be taken to be directional derivative operators. So I need to review this; I shall give rather more detail than Bricker does.

(i) First, the set of spatial or spacetime points is assumed to form a manifold $\mathcal{M}$. The definition of 'manifold' is elaborate, and was only given in its modern formal guise in the 1930s - and fortunately I can skip it! It suffices to say that the definition gives sense to various crucial ideas such as the dimension of a manifold, its boundary (if any), its global topological structure (e.g. being simply connected), the idea of a smooth scalar function i.e. a smooth real-valued function defined on a subset of the manifold-and most important for us, the idea of a smooth curve in the manifold, which is taken as a map $q$ from an interval of real numbers $I \subset \mathbb{R}$ to $\mathcal{M}$. (Here 'smooth' refers to differentiability a specified number of times.) As I said in Section 3.3, the pointilliste will be hard pressed to account for this manifold structure: but I will not labour this point.

(ii) Any curve $q$ thus includes in its definition its real-number parameter, $\lambda$ say. So, understanding the tangent vector to the curve at the point $q(t), t \in I$, in an intuitive way: the tangent vector specifies a directional derivative of any scalar function $f$ defined on a neighbourhood, $N$ say, of the point $q(t), f: N \subset \mathcal{M} \rightarrow \mathbb{R}$. (For the direction of the curve at $q(t)$, together with the "rate at which $\lambda$ ticks away", defines an "instantaneous rate of change" of $f$.)

(iii) It is convenient to identify the tangent vector to the curve $q$ at the point $q(t)$ with the directional derivative operator acting on the set, $\mathcal{R}$ say, 
of all scalar functions defined on some neighbourhood of the point $q(t)$ : $\left.\frac{d}{d \lambda}\right|_{q(t)}:\left.f \in \mathcal{R} \mapsto \frac{d f}{d \lambda}\right|_{q(t)} \in \mathbb{R}$.

Why is it convenient? In short: because the directional derivative operators behave just like tangent vectors. For example, for an $n$-dimensional manifold $\mathcal{M}$, the directional derivative operators at any point $p \in \mathcal{M}$ form an $n$-dimensional vector space, just as one would want the tangent vectors to do: think of the 2-dimensional tangent plane at a point $p$ on the surface of a sphere. This vector space is called the tangent space at $p, T_{p}$.

(Other equivalent identifications are also used: some presentations identify a tangent vector at $p \in \mathcal{M}$ with an equivalence class of curves through $p$-intuitively, curves that are all tangent to each other at $p$ and with parameters "ticking" at the same rate.)

(iv): To define the length of a curve requires still further structure: structure which is not fixed by the postulation of a manifold, with all its tangent vectors $V \in T_{p}$ at each point $p$. Namely, it requires a metric tensor $g$, which is an assignment to each point in $p \in \mathcal{M}$ of a mapping from pairs of vectors $\langle U, V\rangle$ with $U, V \in T_{p}$ to $\mathbb{R}$ : a mapping of a certain sort that generalizes the elementary scalar product of vectors. So $g:\langle U, V\rangle \mapsto g(\langle U, V\rangle) \in \mathbb{R}$. This metric tensor applied to the pair $\langle V, V\rangle$, where $V$ is the tangent vector to a curve $q$ passing through $p$, gives in effect the squared length of the "infinitesimal part" of the curve at $p$. Now, if we let $p$ vary from one point of the curve to another and add up the corresponding contributions, we are performing an integration. So integrating (the square-root of) $g(\langle V, V\rangle)$ gives the length of the curve. One can prove that (as one would want) the length of a curve depends on the metric tensor used, but not on how the curve is parameterized.

To connect (i)-(iv) with Bricker's claim (MetrExtr), one needs some "bridge-principles" between the mathematical constructions and philosophical notions such as that of an intrinsic property. For this, Bricker proceeds as follows. He defines (1993, p. 289) a property $P$ of points to be local iff for any points $p, q$, any neighbourhood $N$ of $p$ and any neighbourhood $M$ of $q$ :

if $N$ is a duplicate of $M$, and $p$ is an $(N, M)$-counterpart of $q$, then $P$ holds either of both $p$ and $q$ or of neither (i.e. $p$ and $q$ match as regards $P$ ). So, roughly speaking, Bricker calls a property $P$ of points 'local' if whether a point $p$ possesses $P$ is wholly determined by the intrinsic nature of any arbitrarily small neighbourhood of $p$. So, modulo the use of metaphysical ideas of intrinsic property, duplicatehood etc., this usage clearly corresponds to mathematicians' use of 'local' (cf. (iii) in Section 2.1.2). 
It follows that for Bricker any intrinsic property of points is local, since counterpart points, being duplicates of each other, share all their intrinsic properties. But, Bricker maintains, the converse fails: there are local but extrinsic properties of points. These he dubs neighbourhood-dependent.

He briefly discusses as examples from elementary calculus, derivatives of functions, in particular instantaneous velocity. He says the instantaneous velocity of a point-particle at position $x$ at time $t$, i.e. at a spacetime point $p$, depends on where the particle is at other times; and so is a neighbourhood-dependent, but not intrinsic, property of $p$. The 'so' here is not spelt out precisely, i.e. by justifying the implicit premise about duplicate spacetime regions containing the particle (or its counterpart). But Bricker's intuition is clear enough: as we emphasised already in Section 2.1.2 and 2.3.2, instantaneous velocity and momentum are temporally extrinsic since for example they imply the object's existence at other times. Besides, the intuition is shared by others - as we will see when we return to instantaneous velocity in the next Section.

Bricker goes on to claim by analogy that in differential geometry all the tangent vectors at a point $p$ 'give information not just about $p$, but about the space immediately surrounding $p .$. in short ... neighbourhood-dependent information about $p$ '. To which he adds: "since the local metric at $p$ is an operator on tangent vectors, it inherits neighbourhood-dependence from its operands" (1993, p. 289).

Again, Bricker's argument here is not entirely precise. He cannot really prove that any property represented by an element of tangent space is extrinsic; for his metaphysical apparatus does not tie its notions of perfectly natural property, and so duplicate, and so $(X, Y)$-counterparthood, sufficiently tightly to the notions of differential geometry. A footnote admits that (as in my (iii) above), tangent vectors are directional derivative operators; but again there is no justification for the implicit premise about duplicate spacetime regions. But fair enough, I say: his intuition is again both clear and shared by others.

And the intuition is enough to deliver Bricker his problem. That is: the metric's being neighbourhood-dependent contradicts the previous claim that it is perfectly natural (i.e. perfectly natural because mentioned in the laws of general relativity) - once we recall that according to his metaphysical framework, all perfectly natural properties are intrinsic.

In response, Bricker considers two tactics for escape from contradiction: an obvious one which he rejects in the second stage of his argument 
(Section 4.4); and an unobvious one which he endorses in the last stage (Section 4.5).

\subsection{Vetoing perfectly natural extrinsics}

Bricker now argues for:

(VetoExtr): The obvious anti-pointilliste response to the contradiction between the metric being neighbourhood-dependent and perfectly natural-viz. that some but not all perfectly natural properties are intrinsic - clashes with the Humean principle of recombination for spacetime points. That is: it engenders necessary connections between a point and its surrounding space.

Bricker first considers saying that some but not all perfectly natural properties are intrinsic. So the idea is that the perfectly natural but extrinsic properties of points include vectorial and tensorial properties, like having a metric tensor with such and such features.

Bricker notes that this response implies that his previous definition of ' $\mathrm{du}$ plicate' bifurcates into a weaker and a stronger notion. The weaker notion is that of intrinsic duplicates: this requires only that the one-one correspondence between the parts of objects $X$ and $Y$ preserve the intrinsic perfectly natural properties and relations. (Recall that Bricker calls a relation 'intrinsic' iff it is internal or external in Lewis' sense, given in Section 3.3.) The stronger notion, which Bricker calls local duplicates, has the same definition, word for word, as the previous definition of duplicates: $X$ and $Y$ are local duplicates iff there is a one-one correspondence between their parts preserving all perfectly natural properties and relations. Bricker proposes that we now define a local property as one that never differs between local duplicates. So it is now built in to the definitions that perfectly natural properties are local—just as previously it was built in that they were intrinsic. Returning to geometry, the idea will be that such perfectly natural, and so local, properties include vectorial and tensorial properties, like having a metric tensor with such and such features.

So far, so good. But there is a clash with Bricker's Humean principle of recombination for points ((iv) of Section 4.2.1): that for any points $p$ and $q$, there is a world whose space is a duplicate of the space of $p$, except that it contains a duplicate of $q$ where the duplicate of $p$ would be. More precisely: Bricker says there is a dilemma.

For this principle must now refer either to (A) local duplicates, or to (B) intrinsic duplicates: and on either interpretation, Bricker sees trouble. I will reply that the second interpretation, (B), is fine-provided one is not a pointilliste. 


\subsubsection{Trouble with local duplicates}

If the principle of recombination refers to local duplicates, then it will yield contradictory worlds when $p$ and $q$ have contrary perfectly natural, extrinsic (but of course local) properties. Bricker gives as his example positive and negative curvature. He writes: "suppose that $p$ is surrounded by positively curved space, $q$ by negatively curved space. Then a world whose space is a duplicate of the space of $p$ but with a local duplicate of $q$ in $p$ 's place must be both positively and negatively curved in the immediate neighbourhood of $q$ " (1993, p. 290; the last phrase of course means 'immediate neighbourhood of the duplicate of $q^{\prime}$ ).

Here I should amplify Bricker's example — and point out a problem raised by it. Given a metric, one can define a scalar function, in the usual mathematical sense of 'scalar' (viz. a function from the manifold $\mathcal{M}$ to $\mathbb{R}$, so that its value at a point $p \in \mathcal{M}$ is the same, independently of any choice of coordinate system), called the scalar curvature $R$, that has the following remarkable property: although it is a scalar, at each point $p$ its value $R(p)$ is a numerical measure of how curved is the geometry in a neighbourhood of $p$. (In fact a metric is sufficient but not necessary to define $R$ : a connection also allows one to define scalar curvature.) So Bricker is no doubt here assuming that:

(i) $p$ and $q$ have positive and negative scalar curvature, respectively, i.e. $R(p)>0$ and $R(q)<0$; (and if we like, we can take him to assume that all points in their respective neighbourhoods have positive and negative scalar curvature);

(ii) the scalar curvature $R$ is perfectly natural but extrinsic: (more precisely, it is a determinable whose determinates, given by specific values $R()=$. etc., are perfectly natural but extrinsic).

Assumption (ii) raises a problem. Hitherto, we have implicitly assumed that scalar functions represent intrinsic properties of points: our worries have concerned only vectorial and tensorial properties. Now we see there is also a gap between:

(a) the mathematical notion of a scalar, which is a matter of how a quantity transforms (viz. trivially: it takes the same value in all coordinate systems); and

(b) the metaphysical idea of intrinsicness.

That is: some scalars can "give information not just about $p$, but about the space immediately surrounding $p$ ”-to quote Bricker's words from his discussion of tangent vectors (quoted in Section 4.3's discussion of Bricker's 
(MetrExtr)). So Bricker owes us a discussion of how exactly being a scalar, and being intrinsic, relate. But this is not to say that the onus is only on Bricker. So far as I know, this is a lacuna in the whole metaphysical literature. (Cf. comment (a) at the end of Section 2.1.2.)

To sum up: the metaphysical literature assumes that any scalar represents an intrinsic property of points, so that the pointilliste need "only" worry whether vectors, tensors etc. do as well. But now we see that pointillistes should also worry about scalars such as the scalar curvature.

\subsubsection{Alleged trouble with intrinsic duplicates}

On the other hand, suppose the principle of recombination refers to intrinsic duplicates. Then contradictory worlds are avoided; but, says Bricker, the principle is now too weak to capture the spirit of the Humean denial of necessary connections between distinct existences. For the principle now rules out necessary connections between the intrinsic natures of distinct objects. But on the present response, an object's "nature" can include more than its intrinsic nature, viz. its perfectly natural extrinsic properties. So, says Bricker, the principle's free combinability of intrinsic natures is not enough to prevent unwanted necessary connections.

I reply that this second horn of Bricker's dilemma has force only for a pointilliste. To see the point, let us take an example. Bricker does not give one: but he could add to the above example of $p$ and $q$, as follows. If one scalar function, say temperature $\theta$, represents an intrinsic property, while the scalar curvature $R$ represents a perfectly natural extrinsic property (and for simplicity, there are no other scalar, vector or tensor functions to consider), the principle of recombination yields a world that has in $p$ 's place an intrinsic duplicate of $q$-i.e. a point with:

(a) the same temperature $\theta$ that $q$ has (in its world), but

(b) the scalar curvature $R$ that $p$ has (in its world), i.e. a positive value, not $q$ 's negative value.

In short, the fact that the neighbourhood of this point is a duplicate of $p$ 's neighbourhood forces the duplicate of $q$ going into $p$ 's place to "shed" its negative scalar curvature.

I take it that Bricker would see this as an unacceptable un-Humean necessary connection between the point $p$ and its surrounding space. But I claim that the wise Humean has no worries here: the necessary connection merely reflects the extrinsicality of scalar curvature, so that the value of the scalar curvature in the surrounding space can constrain its value in $p$ 's 
place. ${ }^{12}$ Besides, I would say that only someone in the grip of pointillisme-an explicit advocate like Lewis, or someone feeling its lure-would be uneasy at having fundamental (if you like, in Lewisian terms: perfectly natural) quantities that are extrinsic to a point. And so much the worse for pointillisme!

But to return to Bricker: he believes that both horns of the dilemma are unacceptable-and so his own preference is ...

\subsection{A heterodox but intrinsic metric}

Bricker thinks we should retain his original metaphysical framework, with its claim that all perfectly natural properties are intrinsic; and we should escape the contradiction at the end of Section 4.3, by giving up the idea that the metric is a perfectly natural property of a point. That is, he proposes:

(Heterodox): We should take the metric tensor to represent an intrinsic property of an infinitesimal neighbourbood of a point, taking such neighbourhoods as genuine mathematical objects.

More precisely, we should hold that the metric, an extrinsic and not perfectly natural property of a point, is "grounded" in another intrinsic, perfectly natural property of a neighbourhood (Bricker's scare-quotes).

Since Bricker presents this preferred solution briefly, and I shall object to it, it is both clearest and fairest to quote him at length. He writes

To illustrate the sort of grounding I have in mind, consider mass density. If one assumes that each neighbourhood of a point has some determinate (finite) mass and volume, then the mass density at a point can be characterized as the limit of the ratio of mass to volume, as volume shrinks to zero. So characterized, mass density is an extrinsic property of points. But it is customary in physics, when considering a continuous matter field, to instead take mass density to be a primitive scalar field: a function that assigns to each point a real number representing (given appropriate units)

12 A mollifying side-remark:- On the other hand, I see no worries, even for Bricker and other advocates of a principle of recombination, lurking in the fact that laws typically require the values of quantities, including scalar intrinsic quantities (such as temperature in my example), to be continuous, or even differentiable a specified number of times. For the principle says only that a world given by recombination is logically possible - not that it obeys the laws of either of the worlds of the "recombined ingredients". 
the intrinsic mass density at the point. Given intrinsic mass density, and an assumption about its smooth distribution, mass can be defined by integration. Extrinsic mass density thus supervenes upon intrinsic mass density. And, thanks to a fundamental theorem of integral calculus, the values of extrinsic and intrinsic mass density coincide .... The suggestion, then, is to say something analogous about the local metric: the extrinsic local metric supervenes on an intrinsic local metric (plus manifold structure).(1993, p. 290-1.)

But, he then says, there is a problem.

How can a tensor be intrinsic to a point? Points are spatially simple. Tensors, being operators on vector spaces, are spatially complex. It is repugnant to the nature of a point to suppose that a local metric, which is a tensor, could be intrinsic to a point ... [the intrinsic local metric] had better be intrinsic not to a point, but to something spatially complex. (1993, p. 291.; with a footnote endorsing Robinson's argument which I reported in Section 4.1, that vectorial properties must be extrinsic to a point.)

He immediately goes on

No sooner said than done. If we are willing to postulate perfectly natural properties on theoretical grounds, we should be willing to posit appropriate entities to instantiate those properties: in this case, entities that are spatially complex. I propose that we reify talk of the "infinitesimal neighbourhood" of a point. The tangent space at a point is now conceived as the infinitesimal neighbourhood of the point "blown large" ... it no longer depends for its existence on the manifold structure. Tensor quantities are intrinsic ... to the infinitesimal neighbourhoods of points... space (or spacetime) has a "non-standard" structure. There are "standard" points, and there are "nonstandard" points that lie an infinitesimal distance from standard points. The points along a path in space are ordered like the nonstandard continuum of Abraham Robinson's non-standard analysis (ibid.)

\subsection{Anti-pointilliste reply}

My reply is clear from what I said in Section 4.4.2. Namely, I think Bricker's principle of recombination is a poor reason for proposing non-standard 
analysis. Though of course non-standard analysis is impressive and fascinating, the fact that vectorial and tensorial properties are extrinsic to a point gives no good reason to adopt non-standard analysis as a metaphysical foundation for differential geometry: only pointillisme makes one think so.

The errors of pointillisme also show up in what Bricker says about his motivating example, mass density; in particular, his saying "it is customary in physics, when considering a continuous matter field, to instead take mass density to be a primitive scalar field".

I reply that this is a mistake. That is: the classical mechanics of continua (whether fluids or deformable solids) conceives mass density exclusively as a limit of a ratio of mass to volume, and so as extrinsic - in just the way Bricker says at the start of the first quotation. And it is right to do so. For use of a primitive mass density scalar field leads to conceptual conundrums. (Agreed, under suitable conditions of smoothness, such a field meshes as regards the mathematics with the usual definition as a limit-as Bricker mentions.)

As a very simple example of such a conundrum, imagine that the unit square $[0,1]^{2} \subset \mathbb{R}^{2}$ is a sheet of continuous material, with a uniform mass density $\rho(x, y)=1$ (so that the total mass is also 1 ). Now suppose the material is expanded to four times its original area, by a uniform stretch, so as to cover the set $[0,2]^{2}$. That is, there is a stretching function $f$ :

$$
f:(x, y) \in[0,1]^{2} \mapsto(2 x, 2 y) \in[0,2]^{2}
$$

The conservation of mass requires that after the expansion $\rho(x, y)=0,25$ for all $(x, y)$. But if as Bricker suggests, the mass density $\rho$ is primitive, it is natural to ask: how does the point-sized bit of matter at a point $(x, y)$ "know" how to decrease its value of $\rho$ between the initial and final times, say $t_{0}$ and $t_{1}: \rho\left(x, y ; t_{1}\right)=\frac{1}{4} \rho\left(x, y ; t_{0}\right)$ ? After all, each point is mapped by $f$ to just one point, not to four points!

On the other hand, there is no such conundrum (in this example, or countless others) if we first state the conservation of mass in terms of extended regions, and then treat mass density as a derived concept.

In fact, we have here come full circle: we have returned to Section 3.3.2's paradox of the length of a line, which launched our discussion of whether pointillisme can accommodate the structure of space or spacetime. For rigorous presentations of continuum mechanics (e.g. Truesdell 1991, pp. 16-19, 92-94) treat mass and mass density in exactly this way. That is: they postulate a mass measure that assigns values of mass to (an appropriate subset 
of all) spatial regions. Mass density is then introduced as a derived concept (essentially the limit of the ratio of mass to volume, as mentioned above), subject to certain conditions that ensure that its integral yields back the original mass-measure for regions. The full details of this treatment require modern measure theory: (for example, the conditions for the density's integral to equal the original measure are given essentially by the RadonNikodym theorem; for details, cf. e.g. Kingman and Taylor (1977, Theorem 6.7)).

But I do not need to rehearse these details: here it is enough to give a non-rigorous statement of how this treatment, applied to the unit square example, avoids the conundrum of how $\rho$ can "know" how to decrease. ${ }^{13}$

In this example, we postulate that all regions $R$ of a suitable kind $\mathcal{K}$ are assigned a mass $m(R, t)$ at a time $t$, which is conserved under the stretching in the sense that

$$
\forall R \in \mathcal{K}, \quad m\left(R, t_{0}\right)=m\left(f(R), t_{1}\right)
$$

We also postulate that each region $R$ is assigned an area $a(R)$; and that the kind $\mathcal{K}$ is rich enough in the sense that for each point $(x, y)$, there is a sequence of regions $\left\{R_{n}\right\}$ which all contain $(x, y)$ but whose areas descend to 0 - which we write as $R_{n} \rightarrow(x, y)$. Then we define the mass density at $(x, y)$ as the corresponding limit of the ratio of mass to area: we assume here that this limit exists, for all $(x, y)$. That is:

$$
\rho(x, y ; t):=\lim _{R_{n} \rightarrow(x, y)} \frac{m(R, t)}{a(R)} .
$$

The conservation of mass, represented fundamentally by eq. 4.2 , can then be re-expressed in terms of the integral of the density

$$
\int_{R} \rho\left(x, y ; t_{0}\right) d x d y=\int_{f(R)} \rho\left(x, y ; t_{1}\right) d x d y
$$

And from this, it follows that $\rho$ must decrease uniformly by a factor of 4 : i.e. $\rho\left(x, y ; t_{0}\right)=4 \rho\left(x, y ; t_{1}\right)$. That is "how $\rho$ knows" how to decrease! ${ }^{14}$

13 I also admit that (as mentioned in Section 1 and 3.3.2) measure theory has some well-nigh paradoxical results of its own. But neither swallowing those results, nor avoiding them by revising measure theory, gives any support to pointillisme.

14 Of course, conundrums like this about the unit square can be formulated not only about mass and mass density, but about arbitrary measures and their densities. And 
Acknowledgements:-I am grateful to audiences at Florence, Kirchberg, Leeds, London, Oxford, and Princeton; and to A. Elga, G. Belot, P. Forrest, J. Hawthorne, S. Leuenberger, L. Lusanna, M. Pauri, H. Price, J. Uffink, and B. van Fraassen for conversations and comments.

\section{References}

Arntzenius, F. 2004 'Gunk, topology and measure', available at: http:// philsciarchive.pitt.edu/archive/00001792.

Arntzenius, F. and Hawthorne, J 2006 'Gunk and continuous variation', forthcoming.

Arthur, R. 2006 'Leibniz's syncategorematic infinitesimals, smooth infinitesimal analysis and Newton's proposition 6', forthcoming.

Batterman, R. 2003 'Falling cats, parallel parking and polarized light', Studies in History and Philosophy of Modern Physics 34B, pp. 527-558.

Bell,J. 1998 A Primer of Infinitesimal Analysis, Cambridge University Press.

Belot, G. 1998 'Understanding electromagnetism', British Journal for the Philosophy of Science 49, pp. 531-555.

Black, R. 2000 'Against Quidditism', Australasian Journal of Philosophy 78, pp.87-104.

Bricker, P. 1993 'The fabric of space: intrinsic vs. extrinsic distance relations', in P. French et al. (eds.), Midwest Studies in Philosophy 18, University of Minnesota Press, pp. 271-294.

Butterfield, J. 2004 'On the Persistence of Homogeneous Matter', available at:physics/0406021: and at http://philsci-archive.pitt.edu/archive/ $00002381 /$

- 2004a 'The Rotating Discs Argument Defeated', forthcoming in British Journal for the Philosophy of Science; available at: http://philsci-archive. pitt.edu/archive/00002382/

- 2005 'On the Persistence of Particles', in Foundations of Physics 35, pp. 233-269, available at: physics/0401112; and http://philsci-archive. pitt.edu/archive/00001586/.

- 2006 'Against Pointillisme about mechanics', forthcoming in British Journal for the Philosophy of Science; available at http://philsci-archive.pitt.edu.

the solution provided by modern measure theory is the same: take the measure, with its assignment to extended regions, as primary, and take the density as a derived concept, viz. as a limit of the ratio of the measure to a volume or area measure. 
- 2006a 'Against Pointillisme: a call to arms', in preparation.

Butterfield, J. and Isham, C. 1999 'On the Emergence of Time in Quantum Gravity', in ed. J Butterfield, The Arguments of Time, British Academy and Oxford University Press, pp. 111-168; available at: gr-qc/ 9901024.

- 2001 'Spacetime and the Philosophical Challenge of Quantum Gravity', in ed.s C. Callender and N. Huggett, Physics meets Philosophy at the Planck Scale, Cambridge University Press pp. 33-89; available at: gr-qc/9903072.

Carathéodory, C. 1963 Algebraic Theory of Measure and Integration, trans. F. Linton, New York: Chelsea Publishing Company.

Davies, E. 2003 'Quantum mechanics does not require the continuity of space', Studies in History and Philosophy of Modern Physics 34B, pp. 319-328.

Earman, J. 1987 'Locality, non-locality and action-at-a-distance: a skeptical review ofsome philosophical dogmas', in Kelvin's Baltimore Lectures and Modern Theoretical Physics, eds. R. Kargon and P. Achinstein, Cambridge Mass: MIT Press.

Earman, J. and Roberts, J.T. 2006 'Contact with the Nomic: a challenge for deniers of Humean supervenience about laws of nature', Philosophy and Phenomenological Research, forthcoming.

Forrest, P. 2002 'Non-classical mereology and its application to sets', The Notre Dame Journal of Formal Logic 43, pp. 79-94.

- 2004 'Grit or gunk: implications of the Banach-Tarski paradox', The Monist 87, pp. 351-384.

Hawthorne, J. 2006 'Quantity in Lewisian Metaphysics', forthcoming in his Metaphysical Essays, Oxford University Press.

Hoefer, C. 2000 'Energy Conservation of in GTR', Studies in History and Philosophy of Modern Physics, 31B pp. 187-200.

Kingman, J. and Taylor, S. 1977 Introducton to Measure and Probability, Cambridge University Press.

Kragh, H. and Carazza, B. 1994 'From time atoms to spacetime quantization: theidea of discreet time 1925-1926', Studies in History and Philosophy of Modern Physics 25, pp. 437-462.

Langton, R. and Lewis, D. 1998 'Defining 'intrinsic", Philosophy and Phenomenological Research 58, pp. 333-345; reprinted in Lewis (1999a), page reference to reprint.

Leibniz, G. 2001 The Labyrinth of the Continuum: writings on the continuum 
problem 1672-1686, ed. sel. and transl. R. Arthur, New Haven: Yale University Press.

Lewis, D. 1983 'Extrinsic properties', Philosophical Studies 44, pp. 197-200; reprinted in Lewis (1999a); page references to reprint.

- 1983a 'New Work for a Theory of Universals', Australasian Journal ofPhilosophy 61, pp. 343-77; reprinted in Lewis (1999a), page reference to reprint.

- 1986 Philosophical Papers, volume II, New York: Oxford University Press.

- 1986a On the Plurality of Worlds, Oxford: Blackwell.

- 1994 'Humean Supervenience Debugged', Mind 103, p 473-490; reprinted in Lewis (1999a), pp. 224-247; page reference to reprint.

- 1999 'Zimmerman and the Spinning sphere', Australasian Journal of Philosophy 77, pp. 209-212.

- 1999a Papers in Metaphysics and Epistemology, Cambridge: University Press.

- 2001 'Redefining 'intrinsic", Philosophy and Phenomenological Research 63, pp. 381-398.

McLarty, C. 1988 'Defining sets as sets of points of spaces', Journal of Philosophical Logic 17, pp. 75-90.

Mancosu, P. 1996 Philosophy of Mathematics and Mathematical Practice in the Seventeenth Century, Oxford: University Press.

Menger, K. 1978 'Topology without points', in Selected papers in Logic and Foundations, Didactics, Economics, Dordrecht: Reidel, pp. 80-107.

Monk, N. 1997 'Conceptions of spacetime: problems and possible solutions, Studies in History and Philosophy of Modern Physics 28, pp. 1-34.

Nelson, E. 1987 Radically Elementary Probability Theory, Princeton University Press.

Oppy, G. 2000 'Humean supervenience?', Philosophical Studies 101 pp. 77105.

Robinson, A. 1996 Non-standard Analysis, Princeton University Press.

Robinson, D. 1989 'Matter, Motion and Humean supervenience', Australasian Journal of Philosophy 67, pp. 394-409.

Roeper, P. 1997 'Region-based topology' Journal of Philosophical Logic 26, pp. 251-309.

Sider, T. 2001 Four-Dimensionalism, Oxford University Press.

Skyrms, B. 1993 'Logical atoms and combinatorial possibility', Journal of Philosophy 90, pp. 219-232. 
Taylor, B. 1993 'On natural properties in metaphysics', Mind 102, pp. 81100.

Truesdell, C. 1991 A First Course in Rational Continuum Mechanics, volume 1; second edition; Academic Press.

Vallentyne, P. 1997 'Intrinsic properties defined', Philosophical Studies 88, pp. 209-219.

Wagon, S. 1985 The Banach-Tarski Paradox, Cambridge: University Press.

Weatherson, B. 2002 'Intrinsic vs. extrinsic properties', Stanford Encyclopedia of Philosophy, http://plato.stanford.edu/intrinsic-extrinsic.

Wilson, M. 1998 'Classical mechanics'; in The Routledge Encyclopedia of Philosophy.

Zimmerman, D. 1998) 'Temporal parts and supervenient causation: the incompatibility of two Humean doctrines', Australasian Journal of Philosophy 76, pp. 265-288. 